\title{
Evolving Morphologies and Controllers for Soft-bodied Multicellular Animats Using Gene Regulatory Networks and Artificial Embryogenesis
}

\author{
Michał Joachimczak \\ Systems Modelling Laboratory, \\ Institute of Oceanology PAS, \\ Sopot, Poland \\ mjoach@gmail.com \\ Rene Doursat \\ Research Group in \\ Biomimetics (GEB), University \\ of Málaga, Spain, \\ Complex Systems Institute \\ Paris lle-de-France (ISC-PIF), \\ CNRS, Paris, France \\ doursat@yahoo.com
}

\author{
Taras Kowaliw \\ Complex Systems Institute \\ Paris lle-de-France (ISC-PIF), \\ CNRS, Paris, France \\ taras@kowaliw.ca \\ Borys Wróbel \\ Systems Modelling Laboratory, \\ IO PAS, Sopot, Poland, \\ Evolutionary Systems Lab, \\ Adam Mickiewicz University , \\ Poznań, Poland \\ Institute for Neuroinformatics, \\ University \& ETH Zurich, \\ Switzerland \\ wrobel@evosys.org
}

\begin{abstract}
We provide a short review of our recent work on the evolution of soft-bodied animats able to swim in a two-dimensional fluid-like environment. Our Artificial Life system, called GReaNs, is based on a model of a gene regulatory network (GRN), encoded in a linear genome, and a model of multicellular development. Animat bodies are created by converting cells to point masses and connecting nearby cells with elastic springs. Outer cells form a skin, an external envelope subjected to fluid drag. Each cell can modify the resting length of the springs attached to it, which creates motion in the animat. We review results that we obtained through two approaches for evolvable control. In the first approach, the spring contractions and extensions follow the sine function. The frequency, phase, and amplitude associated with a particular spring are determined at the end of development, by the state of the GRN in the two cells controlling the spring. The parameters remain fixed as the animat moves. In the second approach, the GRN controls the cell's springs in real time, and cells can also communicate through diffusive signals. Various locomotion modes based on diverse morphologies emerge in both setups, including undulation and the use of primitive appendages.
\end{abstract}

Permission to make digital or hard copies of all or part of this work for personal or classroom use is granted without fee provided that copies are not made or distributed for profit or commercial advantage and that copies bear this notice and the full citation on the first page. To copy otherwise, to republish, to post on servers or to redistribute to lists, requires prior specific permission and/or a fee.

GECCO'12 Companion, July 7-11, 2012, Philadelphia, PA, USA

Copyright 2012 ACM 978-1-4503-1178-6/12/07 ...\$10.00.

\section{Categories and Subject Descriptors}

I.2.8 [Artificial Intelligence]: Problem Solving, Control Methods, and Search-Heuristic methods; F.1.1 [Computation by Abstract Devices]: Models of ComputationSelf-modifying machines; J.3 [Life and medical sciences]: Biology and genetics

\section{General Terms}

Algorithms, Design, Experimentation

\section{Keywords}

Artificial Embryogenesis, Body-brain co-evolution, Development, Evolution of locomotion, Evolution of morphology, Gene Regulatory Networks, Generative and Developmental Systems, Genetic Algorithm, Soft-body animats

\section{INTRODUCTION}

The main objective of evolutionary and developmental robotics is to harness biologically inspired mechanisms toward the automatic design of novel structures and controls of robots. In this extended abstract we provide a short review of our recent work on co-evolving the morphology and control of swimming soft-bodied animats $[6,2]$. The approach we follow is based on the evolution of artificial gene regulatory networks (GRNs) that control the development of multicellular structures. Development starts from a single cell and proceeds by cell division and differentiation. We have previously shown that our platform, named GReaNs for Gene Regulatory evolving artificial Networks, enables the evolutionary development of 2D and 3D multicellular structures with asymmetric shapes [3] and patterning [5]. The 


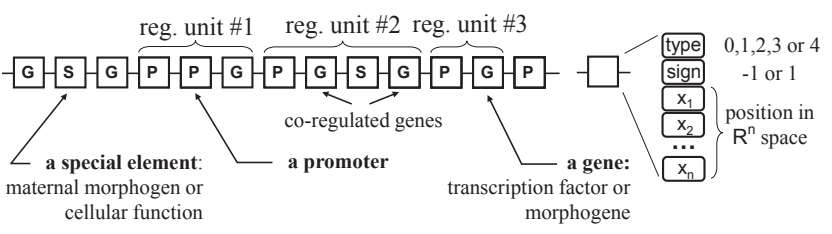

Figure 1: The genome and the genetic elements in GReaNs. Each element consists of a type field, which specifies the class of the element ( $G, P$ or $\mathrm{S})$, a sign field, and a sequence of $N$ abstract coordinates in $\mathbb{R}^{N}$ space $(N=2$ here), which determine its affinity to other elements.

recent extension that we brought to GReaNs allows the conversion of a multicellular structure into a soft-bodied animat. We discuss two different approaches for evolving the control of animat movement, both successful in generating a wide range of morphologies and motion types.

\section{GRN AND EMBRYOGENESIS}

We employ the genomic model and multicellular embryogenesis process that were previously included in GReaNs for controlling the development and differentiation of cells in 3D [5]. Genomes are lists of genetic elements. A genetic element can be a promoter (a regulatory element) or a gene (genes encode products). A series of promoters followed by a series of genes is a regulatory unit. Units form nodes in the GRN (Fig. 1). Connectivity between the nodes is determined by product-promoter affinity. Each genetic element (Fig. 1, right) consists of fields. Each field is a number. One field determines the type of the element. Two fields are the coordinates of a point in an abstract $\mathbb{R}^{2}$ space (not to be confused with the $2 \mathrm{D}$ physical, or virtual, environment). The product-promoter affinity is a function of Euclidean distance between the corresponding points. There is one more field, the sign. It determines whether the interaction is excitatory (the signs of two elements are the same) or inhibitory (the signs differ). Special genetic elements correspond to the GRN's inputs (e.g, one element specifies a product that has a constant concentration) and outputs. Product concentrations change continuously with time and are represented as real values between 0 and 1 . Whether the concentration of the products coded by a particular regulatory unit increases or decreases in the next simulation step depends on the concentration of the products that have an affinity to the promoters of this unit.

The developmental process starts from a single cell. All cells have the same GRN, but the concentrations of the products may differ. These concentrations control the cells' developmental behavior. Cells are displayed as circles. They divide in a simulated fluid-like environment. Cells can produce diffusive products (morphogens), which can in turn influence the behavior of other cells. They can also sense the concentration of substances (maternal morphogens) present in the environment at various concentrations in different locations.

\section{SOFT-BODIED ANIMATS}

Once a multicellular structure is formed, it is converted to a soft-bodied animat (Fig. 2). First, the connectivity between nearby cells is determined using a Gabriel graph [1] (a

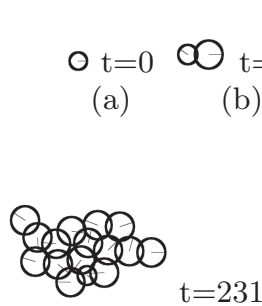

(e)

(b)

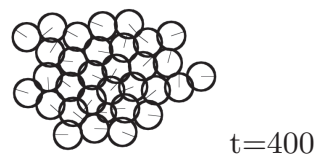

(f) (d)

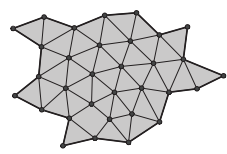

(g)
Figure 2: Development of a multicellular structure and its conversion to a soft-bodied animat. In (af) cells are represented as circles, lines correspond to the direction of division, (g) shows the final structure after connecting cell centers with elastic springs.

subgraph of the Delaunay-Voronoi triangulation preserving concavity). Then, edges of the Gabriel graph are converted to springs (each spring having the same elasticity and damping parameters), vertices of the graph to cell masses (each cell having the same mass), and polygons formed by the edges to pressurized chambers. The outmost edges form the skin. Each cell can contract or expand the springs that are connected to it, provoking changes in the chambers' surface area around this cell. The chambers act as the animat's "hydrostatic skeleton". An animat moves in an environment with fluid drag (using a model described in [8]; the same model was used in [7]). This model assumes that the fluid is stationary and that the force acting on a single edge of the skin is a sum of tangential and normal drag components with respect to the motion of this edge.

\section{TWO APPROACHES TO CONTROL OF LOCOMOTION}

In two recent papers $[6,2]$ we have extended the GReaNs platform to evolve the body and control of soft-bodied animats. In both cases evolution is modeled with a genetic algorithm. The fitness function rewards the distance traveled by the animat's center of mass during a fixed duration. The development and transformation of the multicellular structure into a soft body is the same. Differences concern the way in which the movement is controlled.

In the first study [6], a spring contracts and expands according to the activity of the two cells that it connects. The effect of both cells is additive, and their activity follows a sine function whose parameters remain fixed during animat motion. The phase, frequency and amplitude of the oscillation are determined by the concentrations of three products at the end of development. These parameters remain fixed during the evaluation of a swimming animat, and the GRN is not active during locomotion. In contrast, the approach described in the second paper [2] makes real-time control of actuation possible by keeping the GRN active. The resting length of the springs is determined by the concentrations of two products (one for contraction, one for expansion) in each of the two connected cells. In this model, the movement is caused by oscillations in cell activity, too, but these oscillations are emergent, not given. The fact that they evolve is not surprising, however, since another previous work [4] demonstrated that oscillatory patterns of GRN activity could evolve in GReaNs. Nonetheless, this second 

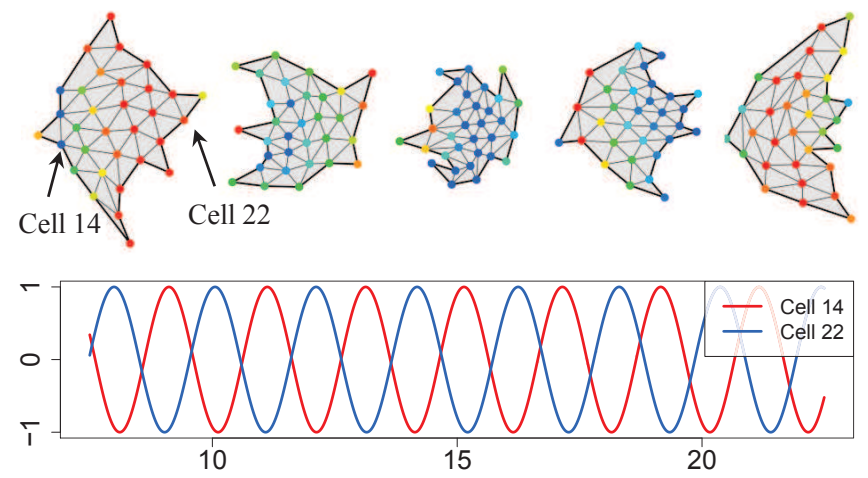

(a)
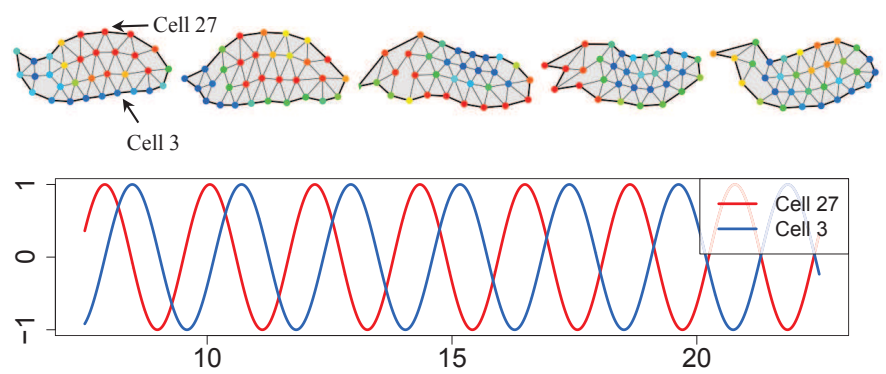

(b)
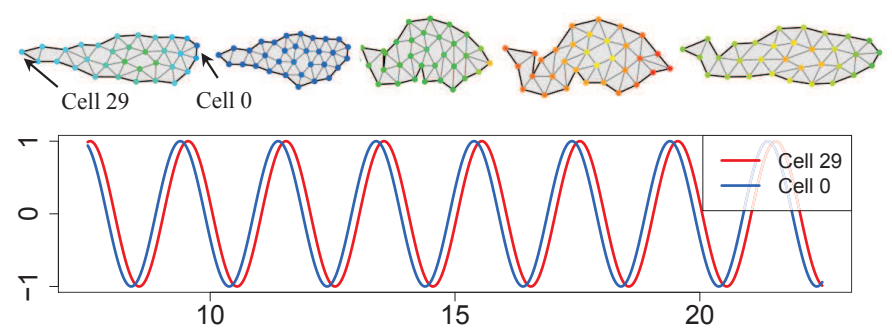

(c)

Figure 3: Examples of individuals which evolved when the parameters of the sine oscillators remained fixed during locomotion. The graphs below each individual show actuation patterns of selected cells over time: 1 corresponds to the maximum signal for expansion of the resting length of an associated spring; -1 to the maximum for contraction. All animats move towards the right.

approach creates a larger search space, opening the possibility to obtain more complex patterns of oscillation.

In both papers we evolved swimming animats under various environmental conditions (the fluid drag in the virtual environment). Both approaches resulted in some of the animats showing similar modes of locomotion and morphology. For example, when the fluid drag was low, evolution often resulted in individuals relying on rudimentary "appendages" (Fig. 3a, 4a), while broader environmental conditions gave rise to undulatory movement (Fig. 3b, 4b). These swimming strategies relied on contracting and expanding all the springs synchronously, with phase shifts along the animat's front-back and/or left-right axes.
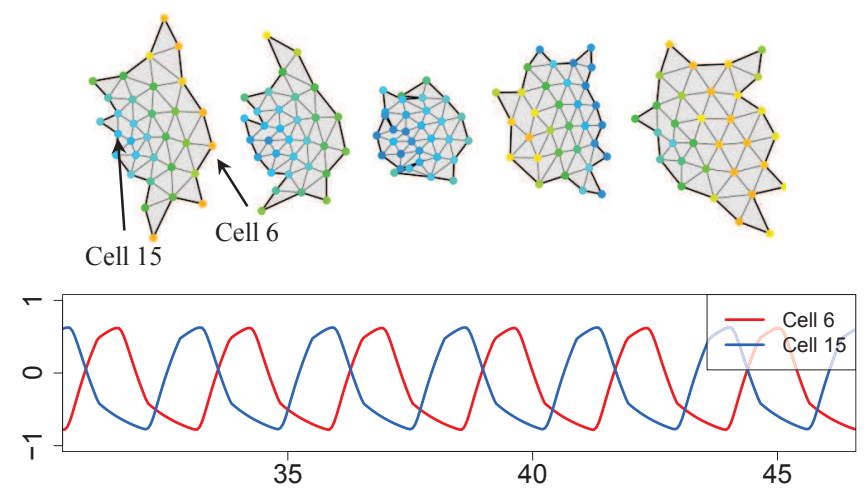

(a)
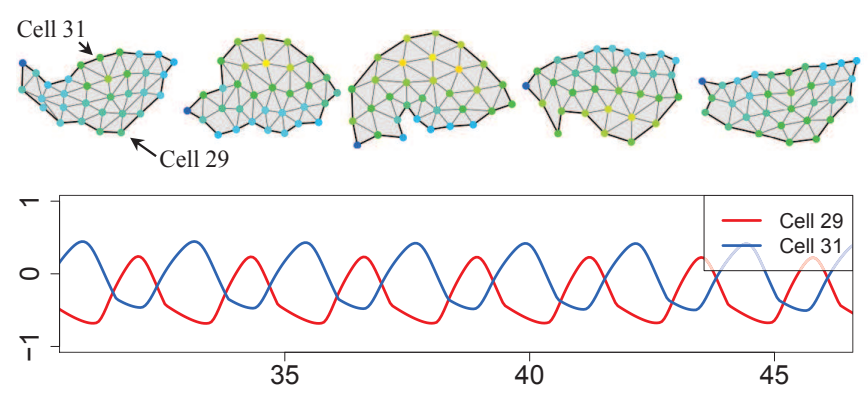

(b)
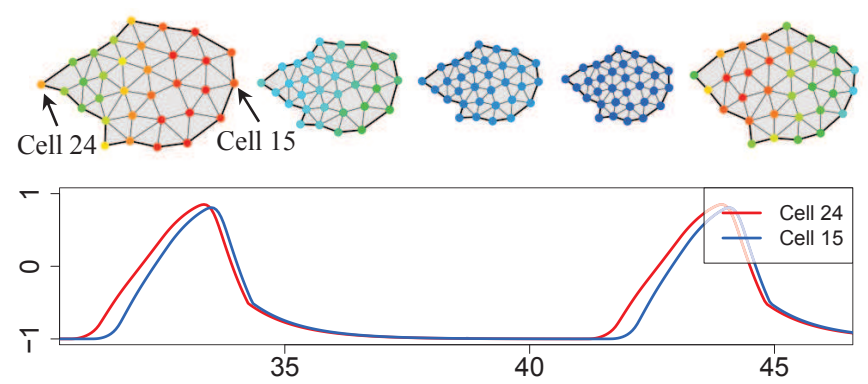

(c)

Figure 4: Examples of individuals which evolved when the GRN remained active during locomotion. See Fig. 3 for details.

When the parameters of the sine oscillators remained fixed, the evolved individuals relied solely on the physical properties of the morphology to generate asymmetric forces during contraction and expansion. One striking example was a "kicking" strategy involving a "tail" that straightened as other springs, closer to the front, made the body contract (Fig. 3c).

In many motion strategies evolved while the GRN remained active during actuation, the resulting patterns of GRN activity approximated a sine function (Fig. 4a, 4b). However, this approach also allowed for the evolution of strategies in which the activity was periodic, but not necessarily sine-like. In effect, we also observed a class of nonsinusoidal individuals, which mostly evolved when the fluid drag was high. These animats exploited the fact that the drag was proportional to the squared velocity of an edge acting against it. Their body was pointed at one end and blunt at the other end, and the speed of expansion differed 
from the speed of contraction (Fig. 4c). Depending on which one was faster, such individuals moved in the direction of the blunt or pointed end.

\section{CONCLUSIONS AND FUTURE WORK}

Our system for the evolution of soft-bodied animats allows for GRNs that are not limited in number of elements or connections. The size of the animats is also not limited in principle (although it was, for computational reasons, in the evolutionary runs reviewed here). In neither approachwhether the GRN was inactive during locomotion or would control it in real time - did we impose restrictions on the exact shape of the animat or the mode of control. This lack of restriction allowed for a wide diversity of morphologies and strategies for locomotion to evolve. Even when allowing the GRN to act during locomotion increased the search space, the system remained evolvable. In this second approach it was possible to observe the evolution of a larger diversity of motion strategies, including a pulsating mode not possible with a sine-like actuation under the first approach.

In both approaches, locomotion control was carried out without a nervous system. The approach in which GRN controls the locomotion in real time is especially interesting in this respect, because in principle it could also enable the active control of the direction of movement, without the need for special elements similar to biological neurons. We envisioned here a mode of control relying entirely on diffusion of cell products from one part of the body to another. In future work, we wish to continue exploring this mode of control, and better understand and assess its limits in comparison to a set-up where some cells differentiate into artificial neurons controlling the animat's behavior.

\section{Acknowledgments}

This work was supported by a PAN-CNRS collaborative project, IO PAN (task IV.3), and a scholarship from the French government to BW. TK's and RD's positions at the ISC-PIF were funded by Région Ile-de-France. Computational resources were provided by the Polish Ministry of Science and Education (project N519 384236, N303 291234), the Tri-City Academic Computer Centre (TASK), and the Interdisciplinary Centre for Molecular and Mathematical Modeling (ICM, University of Warsaw; project G33-8).

\section{References}

[1] K. R. Gabriel and R. R. Sokal. A new statistical approach to geographic variation analysis. Syst. Zool., 18(3):259-278, 1969.

[2] M. Joachimczak, T. Kowaliw, R. Doursat, and B. Wróbel. Brainless bodies: Controlling the development and behavior of multicellular animats by gene regulation and diffusive signals. In Artificial Life XIII: Proceedings of the 13th International Conference on the Simulation and Synthesis of Living Systems. MIT Press, Cambridge, MA, 2012. (submitted).

[3] M. Joachimczak and B. Wróbel. Evo-devo in silico: a model of a gene network regulating multicellular development in 3D space with artificial physics. In Artificial Life XI: Proceedings of the 11th International Conference on the Simulation and Synthesis of Living Systems, pages 297-304. MIT Press, Cambridge, MA, 2008.

[4] M. Joachimczak and B. Wróbel. Processing signals with evolving artificial gene regulatory networks. In Artificial Life XII: Proceedings of the 12th International Conference on the Simulation and Synthesis of Living Systems, pages 203-210. MIT Press, Cambridge, MA, 2010.

[5] M. Joachimczak and B. Wróbel. Evolution of the morphology and patterning of artificial embryos: scaling the tricolour problem to the third dimension. In ECAL 2009: Proceedings of the 10th European Conference on Artificial Life, volume 5777 of Lecture Notes in Computer Science, pages 33-41. Springer, 2011.

[6] M. Joachimczak and B. Wróbel. Co-evolution of morphology and control of soft-bodied multicellular animats. In GECCO '12: Proceedings of the 14th Annual Conference on Genetic and Evolutionary Computation. ACM, 2012. (in print).

[7] L. Schramm, Y. Jin, and B. Sendhoff. Emerged coupling of motor control and morphological development in evolution of multi-cellular animats. In Proceedings of the 10th European Conference on Artificial Life (ECAL2009), volume 5777 of Lecture Notes in Computer Science, pages 18-26. Springer, 2011.

[8] M. Sfakiotakis and D. P. Tsakiris. Simuun : A simulation environment for undulatory locomotion. International Journal of Modelling and Simulation, 26:350-358, 2006. 